\title{
Direct measurement of unsteady microscale Stokes flow using optically driven microspheres
}

\author{
Nicolas Bruot $\odot,{ }^{1,2}$ Pietro Cicuta $\odot,{ }^{1}$ Hermes Bloomfield-Gadêlha $\odot,{ }^{3}$ Raymond E. Goldstein $\odot,{ }^{4, *}$ \\ Jurij Kotar $\odot,{ }^{1}$ Eric Lauga $\odot,{ }^{4}$ and François Nadal $\odot^{5, \dagger}$ \\ ${ }^{1}$ Cavendish Laboratory and Nanoscience Centre, University of Cambridge, \\ Cambridge CB3 OHE, United Kingdom \\ ${ }^{2}$ Institut de Physique de Nice, CNRS, UMR No. 7010, Université Côte d'Azur, 06108 Nice, France \\ ${ }^{3}$ Department of Engineering Mathematics \& Bristol Robotics Laboratory, University of Bristol, \\ Bristol BS8 1UB, United Kingdom \\ ${ }^{4}$ Department of Applied Mathematics and Theoretical Physics, Centre for Mathematical Sciences, \\ University of Cambridge, Cambridge CB3 OWA, United Kingdom \\ ${ }^{5}$ Wolfson School of Mechanical, Electrical and Manufacturing Engineering, Loughborough University, \\ Loughborough LE11 3TU, United Kingdom
}

(Received 28 October 2020; accepted 11 May 2021; published 27 May 2021)

\begin{abstract}
A growing body of work on the dynamics of eukaryotic flagella has noted that their oscillation frequencies are sufficiently high that the viscous penetration depth of unsteady Stokes flow is comparable to the scales over which flagella synchronize. Incorporating these effects into theories of synchronization requires an understanding of the global unsteady flows around oscillating bodies. Yet, there has been no precise experimental test on the microscale of the most basic aspects of such unsteady Stokes flow: the orbits of passive tracers and the position-dependent phase lag between the oscillating response of the fluid at a distant point and that of the driving particle. Here, we report the first such direct Lagrangian measurement of this unsteady flow. The method uses an array of 30 submicron tracer particles positioned by a time-shared optical trap at a range of distances and angular positions with respect to a larger, central particle, which is then driven by an oscillating optical trap at frequencies up to $400 \mathrm{~Hz}$. In this microscale regime, the tracer dynamics is considerably simplified by the smallness of both inertial effects on particle motion and finite-frequency corrections to the Stokes drag law. The tracers are found to display elliptical Lissajous figures whose orientation and geometry are in agreement with a low-frequency expansion of the underlying dynamics, and the experimental phase shift between motion parallel and orthogonal to the oscillation axis exhibits a predicted scaling form in distance and angle. Possible implications of these results for synchronization dynamics are discussed.
\end{abstract}

DOI: 10.1103/PhysRevFluids.6.053102

\footnotetext{
*R.E.Goldstein@damtp.cam.ac.uk

†F.R.Nadal@lboro.ac.uk
}

Published by the American Physical Society under the terms of the Creative Commons Attribution 4.0 International license. Further distribution of this work must maintain attribution to the author(s) and the published article's title, journal citation, and DOI. 


\section{INTRODUCTION}

In his landmark 1851 paper on viscous fluids [1], George Gabriel Stokes not only developed the theoretical framework for understanding the competition between inertial and viscous forces, but he also considered several physical situations in which that competition is particularly simple to analyze. These include his celebrated problems I and II-viscous fluid in the half space adjacent to a no-slip wall that is impulsively started into motion or oscillated from side to side at some frequency $\omega$-as well as the case of a sphere oscillated back and forth. From these oscillatory cases and his newly identified "index of friction" (what we now term the kinematic viscosity $v=\eta / \rho_{f}, \eta$ and $\rho_{f}$ being the dynamic viscosity and density of the fluid), he identified from the diffusion equation $u_{t}=v u_{x x}$, for a component $u$ of the fluid velocity, the viscous penetration length

$$
\delta=(2 v / \omega)^{1 / 2}
$$

as the distance over which oscillatory motions decay away from the driving surface. Furthermore, the fluid oscillations at some distance $r$ from the driving body are phase shifted relative to the drive by an angle proportional to $r / \delta$.

There is, of course, no doubt of the validity of his analysis of these particular problems. Yet, in the motivating biophysical context we consider here there has been longstanding uncertainty about the relevance of unsteadiness to phenomena that are strongly in the Stokes regime, such as the beating of eukaryotic flagella and the motion of tracer particles in flows driven by flagellated organisms. For example, in models for ciliate propulsion [2], it has been recognized that around large microorganisms covered in a dense cilia carpet, unsteady effects are significant within a region near the organism surface of width $\sim \delta$, and outside the flow may be considered steady. Consider for example the well-studied multicellular organism alga Volvox [3], a spheroid of radius $\sim 200 \mu \mathrm{m}$, covered with thousands of biflagellated somatic cells each $10 \mu \mathrm{m}$ in diameter, spaced some $20 \mu \mathrm{m}$ apart, whose flagella of length $\ell \sim 25 \mu \mathrm{m}$ beat at a frequency $f \sim 25 \mathrm{~Hz}$. The viscous penetration depth $\delta \sim 110 \mu \mathrm{m}$ is significantly less than the organism's circumference, but it is intriguingly close to the wavelength of metachronal waves that Volvox exhibits [4,5]. These are long-wavelength phase modulations of the beating in the form like that of a stadium wave which, in Volvox, have a wavelength $\sim 100 \mu \mathrm{m}$ [5]. Thus, even nearest-neighbor somatic cells have a significant phase shift. This is in contrast to the situation in Chlamydomonas, the unicellular relative of Volvox whose size is comparable to Volvox somatic cell and whose two 10-12 $\mu \mathrm{m}$ flagella are mounted just a few microns apart and beat at $\sim 50 \mathrm{~Hz}$; the phase shift between the flagella is indeed rather small. Yet, nearly all models of flagellar synchronization and in particular of metachronal wave formation [6-14] assume as a starting point the steady Stokes equation. It is only recently, in the context of the dynamics of tracer particles in flows generated by the beating flagella of alga [15-18] that unsteadiness has been identified as a potentially significant feature of biophysical flows.

With the goal of motivating further studies of these phenomena, we introduce an experimental setup by which optical trapping methods $[19,20]$ are used to measure the motion induced by unsteadiness over a broad angular sector around a central oscillated microsphere. This setup allows for a precise test of the underlying microhydrodynamic theory, with results that are complementary to recent experimental studies of oscillatory flows driven by the more complex beating of Chlamydomonas flagella, where the phase lag experienced by tracer particles was measured directly $[16,18]$. As shown in earlier work on synchronization [21], the far-field flows due to eukaryotic flagella are accurately represented by moving point forces. Thus, we expect the present results to inform future analysis of flagellar interactions on the basis of simplified representations of their dynamics.

We begin in Sec. II with a description of the experimental setup, the frequency response of the optical trap used to oscillate a microsphere surrounded by an array of tracers, and a discussion of inertial corrections to the motion of the microspheres. The results presented in Sec. III comprise the motion of tracer particles at varying distances and angular positions relative to the driven microsphere. The theoretical analysis of their Lagrangian dynamics is done with the Eulerian flow field of the classical solution for motion around an oscillating sphere. A low-frequency expansion, 


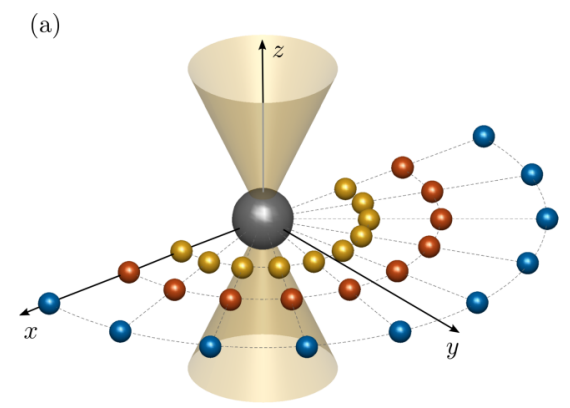

(b)

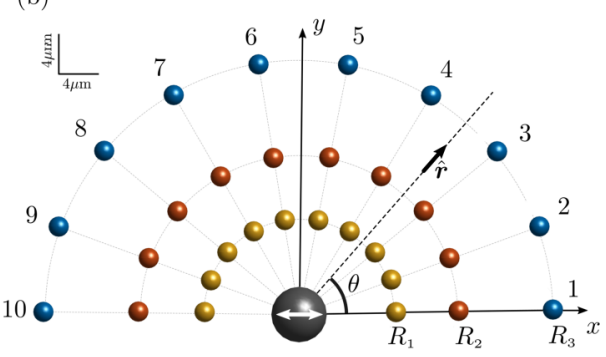

FIG. 1. Schematic of the experiment. (a) Side view. The large silica microsphere (gray) of radius $a_{0}=$ $2.77 \mu \mathrm{m}$ is oscillated at various frequencies by means of an optical trap. Previous to the actuation of the large particle, passive microspheres (yellow, red, blue) with radius $a_{1}=0.505 \mu \mathrm{m}$ are placed at three different distances $R_{1}=9.51 \mu \mathrm{m}, R_{2}=15.85 \mu \mathrm{m}$, and $R_{3}=25.36 \mu \mathrm{m}$ from the center of the driving bead. They are held in position via a multiple trap system, and released automatically upon actuation of the central sphere, whose motion along the $x$ axis is shown by a double white arrow in top view (b).

valid when $\delta$ is large compared to distances from the central sphere, is used to obtain a geometrically simple result for the tracer trajectories, which are elliptical Lissajous figures. As the tracers are submicron, they exhibit substantial thermal fluctuations which compete with the deterministic displacements from the oscillating flow, and this can be quantified by a suitable Péclet number that varies with oscillation frequency and distance from the central particle. The phase shift between motion along the two Cartesian directions, which is responsible for the shape of those orbits, is calculated in the low-frequency limit and found to be in excellent agreement with the data. The implications of the observed phase shift between the driven and tracer particles on synchronization processes relevant to the biomechanics of cilia are discussed in the concluding Sec. IV.

\section{EXPERIMENTAL SETUP}

\section{A. Optical trapping}

A large silica microsphere (Bangs Laboratories, mean radius $a_{0} \sim 2.77 \mu \mathrm{m}$, coefficient of variation $\sim 10 \%, \rho_{0}=2.65 \times 10^{3} \mathrm{~kg} \mathrm{~m}^{-3}$ ) is forced to oscillate horizontally along the $x$-axis with an amplitude $\xi_{0}(t)=\xi_{0} \exp (\mathrm{i} \omega t)$ in water (density $\rho_{w}=10^{3} \mathrm{~kg} \mathrm{~m}^{-3}$, viscosity $\eta=10^{-3}$ Pas) by means of optical tweezers. As we are dealing with linear problems in this paper, here and below it is the real part of complex expression that corresponds to the physically observable quantities. Smaller passive polystyrene microspheres (Polyscience, mean radius $a_{1}=0.505 \mu \mathrm{m}, \mathrm{CV} \sim 5 \%$, density $\rho_{1}=1.05 \times 10^{3} \mathrm{~kg} \mathrm{~m}^{-3}$ ), also referred to as probes or tracers, are located in the horizontal $(x, y)$ plane at three different distances $R_{i}\left(R_{1}=9.51 \mu \mathrm{m}, R_{2}=15.85 \mu \mathrm{m}\right.$ and $\left.R_{3}=25.36 \mu \mathrm{m}\right)$ from the central sphere, and ten different angles $\theta_{j}(j=1 \cdots 10)$ equally spaced within the interval $[0, \pi]$, as shown in Fig. 1 . The Lagrangian displacement of a polystyrene sphere located at $\left(R_{i}, \theta_{j}\right)$ due to the flow generated by the central bead is denoted by $\xi^{i j}=\left(\xi_{x}^{i j}, \xi_{y}^{i j}\right)$.

The particles used in each experimental run were extracted from dilute suspensions of microspheres. The polystyrene and silica beads were sufficiently dilute that no particles other than those used as oscillator or probes interfered with the laser beam during an experiment. The solution was sealed between a microscope slide and a coverslip separated by a $150 \mu \mathrm{m}$ gap and held together by NOA $68 \mathrm{UV}$-cured glue. Microspheres were trapped at least $50 \mu \mathrm{m}$ from the chamber walls to minimize any wall-particle interactions.

The tweezers setup is as described elsewhere [14,22,23]. Briefly, the beam of a diode-pumped solid-state laser (CrystaLaser IRCL-2W-1064, $1064 \mathrm{~nm}$ wavelength, $2 \mathrm{~W}$ maximum output power) is deflected by a pair of acousto-optic deflectors (AA.DTS.XY-250@1064 nm, AA Opto-Electronic) and directed to the back illumination port of a Nikon Ti-E inverted microscope, in which the beam 
is reflected by a dichroic mirror and focused on the sample by a Nikon Plan Apo VC 60x WI objective $(\mathrm{NA}=1.20)$. The samples were viewed with brightfield illumination and the dynamical response of the microspheres to the oscillating flow was recorded by high-speed camera (Phantom V5.1) at 25,000 frames per second. The acousto-optic deflector allows for time-sharing of the laser beam so that multiple traps located at prescribed positions can be created in the $(x, y)$ focal plane. The stiffness of the trap for the silica when it is trapped alone was determined to be $k=50 \pm$ $1 \mathrm{pN} \mu \mathrm{m}^{-1}$, by measuring the standard deviation of the particle's thermal fluctuations.

Initially, the silica bead is trapped at the origin of the coordinate system while the ten polystyrene particles are held at locations $\left(R_{i}, \theta_{j}\right)$, one $R_{i}$ at a time. The central particle is driven by moving its optical trap along the $x$ axis by sampling the path as $N_{p}$ points which are cyclically visited by the acousto-optic deflector. Once the polystyrene particles are released, the entire laser power is reassigned to the oscillating trap and the trajectories of the silica and polystyrene particles are recorded. The corresponding tracks in the $(x, y)$ plane are extracted using a bespoke image segmentation tracking algorithm. To suppress the effect of thermal fluctuations of the tracers on single oscillations, all the cycles were averaged into a composite, cyclic $x, y$ path for each tracer. Each is obtained by computing the average $x$ and $y$ oscillations as a histogram with $2 \pi f_{s} / \omega$ bins where $f_{s}$ is the sampling frequency; these data are accumulated in the bins using the time $t \bmod (2 \pi / \omega)$. The system was optimized to reach driving frequencies up to $400 \mathrm{~Hz}$ for a trap oscillation amplitude of $2.15 \mu \mathrm{m}$. The oscillation frequency and amplitude are limited by the dynamics of the particle (see below) and the finite size of the optical potential well. Once they are optimized, the maximal $N_{p}$ for path sampling is obtained from the highest target frequency and a time-sharing frequency of $20 \mathrm{kHz}$ set by the bespoke electronics that control the acousto-optic deflector; $N_{p}=50$ in experiments reported here. Under such conditions, the drive bead follows a sinusoidal pattern, even when it does not remain close to the trap center.

\section{B. Microsphere motion}

The dynamics of a microsphere forced by an optical trap whose position is laterally oscillated is a well-studied problem [24]. When, as is the case here, the displacement of the driven particle is sufficiently small that the optical force exerted on the sphere is linearly proportional to the distance from the axis of the beam, and the trap and particle positions oscillate as $\zeta_{0} \mathrm{e}^{i \omega t}$ and $\zeta_{\text {trap }} \mathrm{e}^{i \omega t}$ (where $\zeta_{0}$ is complex, reflecting the phase shift of the particle relative to the trap), then momentum conservation in the $x$ direction takes the form

$$
\left(-\rho_{0} \mathcal{V}_{0} \omega^{2}+6 \pi \eta a_{0} \Omega_{0} \mathrm{i} \omega\right) \zeta_{0}=-k\left(\zeta_{0}-\zeta_{\text {trap }}\right),
$$

where the left-hand side represents the inertia of the particle itself (whose volume is $\mathcal{V}_{0}=4 \pi a_{0}^{3} / 3$ ) and the drag force, while the right-hand side is the trap force. The drag force, found in the original derivation by Stokes in 1851 [1] and in more modern treatments [25,26], has a factor $\Omega_{0}$ that corrects the familiar zero Reynolds number Stokes drag for fluid inertia,

$$
\Omega_{0}=1+\alpha_{0}+\frac{\alpha_{0}^{2}}{9}, \text { with } \alpha_{0}=(1+i) \frac{a_{0}}{\delta} .
$$

From Eqs. (2) and (3), we identify three characteristic times scales associated with the experiment, the shortest of which is that for fluid momentum to diffuse on the scale of particle,

$$
\tau_{d}=\frac{a_{0}^{2}}{v} \sim 8 \times 10^{-6} \mathrm{~s}
$$

Next is that for inertial oscillations of the sphere in the trap,

$$
\tau_{i}=\left(\frac{\rho_{0} \mathcal{V}_{0}}{k}\right)^{1 / 2} \sim 7 \times 10^{-5} \mathrm{~s}
$$



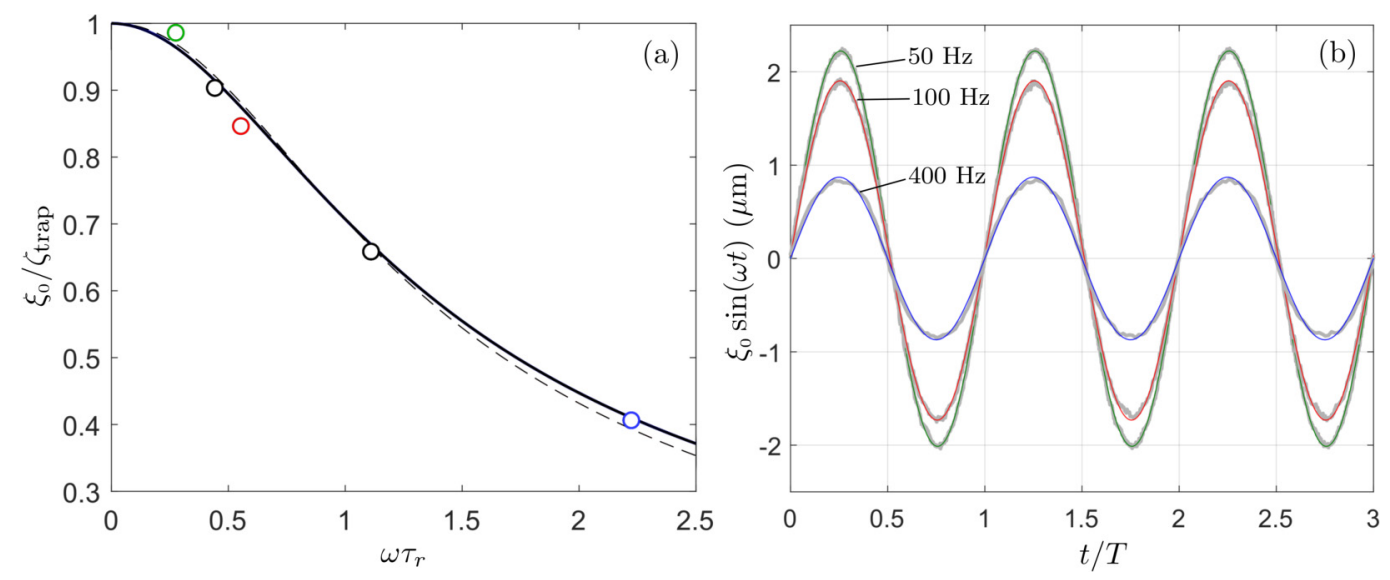

FIG. 2. Oscillations of the driven microsphere. (a) Amplitude of oscillations relative to trap oscillation amplitude as a function of frequency. The theoretical prediction Eq. (9) with $\tau_{d}=0$ (solid line) matches well the experimental data (open circles). Dashed line indicates small correction obtained by including finite $\tau_{d}$ corrections. (b) Measured $x$ position of the bead as a function of the time (thick gray lines), fitted with a sinusoidal function (colored lines) at three frequencies. For clarity of presentation, the origin of time has been shifted to align the different curves. The good match between the experimental profiles and the fitting functions validates the linear approach used to derive Eq. (9).

and finally the timescale over which a particle viscously relaxes to the trap center,

$$
\tau_{r}=\frac{6 \pi \eta a_{0}}{k} \sim 10^{-3} \mathrm{~s}
$$

With these definitions, we have

$$
\zeta_{0}=\frac{\zeta_{\text {trap }}}{1+i \omega \tau_{r} \Omega_{0}-\left(\omega \tau_{i}\right)^{2}}
$$

At the highest frequencies probed $(400 \mathrm{~Hz}) \omega \tau_{i} \sim 0.18$; since this quantity only enters through its square $\left[\left(\omega \tau_{i}\right)^{2} \sim 0.03\right]$, particle inertia contributes only a few percent to the response of microspheres and may be neglected in the denominator of Eq. (7). The dependence of $\Omega_{0}$ on frequency in (2) is through the factor $\alpha_{0}=((1+i) / \sqrt{2})\left(\omega \tau_{d}\right)^{1 / 2}$. The maximum contribution of momentum diffusion has $\omega \tau_{d} \sim 0.02$, so $\left|\alpha_{0}\right| \sim 0.14$, yielding a modest correction to the force amplitude $\Omega_{0}$, and higher-order contributions are negligible. By the quadratic scaling of $\tau_{d}$ with sphere radius, this simplification is due to the use of microspheres. Thus neglecting particle inertia (setting $\tau_{i}=0$ ) and quadratic terms in $\alpha_{0}$ in $\Omega_{0}$, we have

$$
\zeta_{0} \simeq \frac{\zeta_{\text {trap }}}{1-\left(\omega \tau_{r}\right)\left(\omega \tau_{d} / 2\right)^{1 / 2}+i \omega \tau_{r}\left(1+\left(\omega \tau_{d} / 2\right)^{1 / 2}\right)} .
$$

The complex structure of Eq. (8) implies that there is a phase shift between the trap and the driven particle, but as we are interested in the response of the tracers relative to the driven microsphere, we ignore that phase shift and use the motion of the driven bead as the time reference in the following and define $\xi_{0}(\omega)=\left|\zeta_{0}\right|$. If we adopt a time origin such that the driven particle's position is $\xi_{0} \cos (\omega t)$, then

$$
\xi_{0}(\omega) \simeq \zeta_{\text {trap }}\left[\left(1-\left(\omega \tau_{r}\right)\left(\omega \tau_{d} / 2\right)^{1 / 2}\right)^{2}+\left(\omega \tau_{r}\right)^{2}\left(1+\left(\omega \tau_{d} / 2\right)^{1 / 2}\right)^{2}\right]^{-1 / 2},
$$

which represents only minor deviations from the Lorentzian form $\left[1+\left(\omega \tau_{r}\right)^{2}\right]^{-1 / 2}$ that holds when $\tau_{d}=0$ (and then $\Omega_{0}=1$ ). This ratio and its Lorentzian approximation are plotted in Fig. 2(a) as a function of the rescaled frequency $\omega \tau_{r}$. With no free parameters the agreement between 

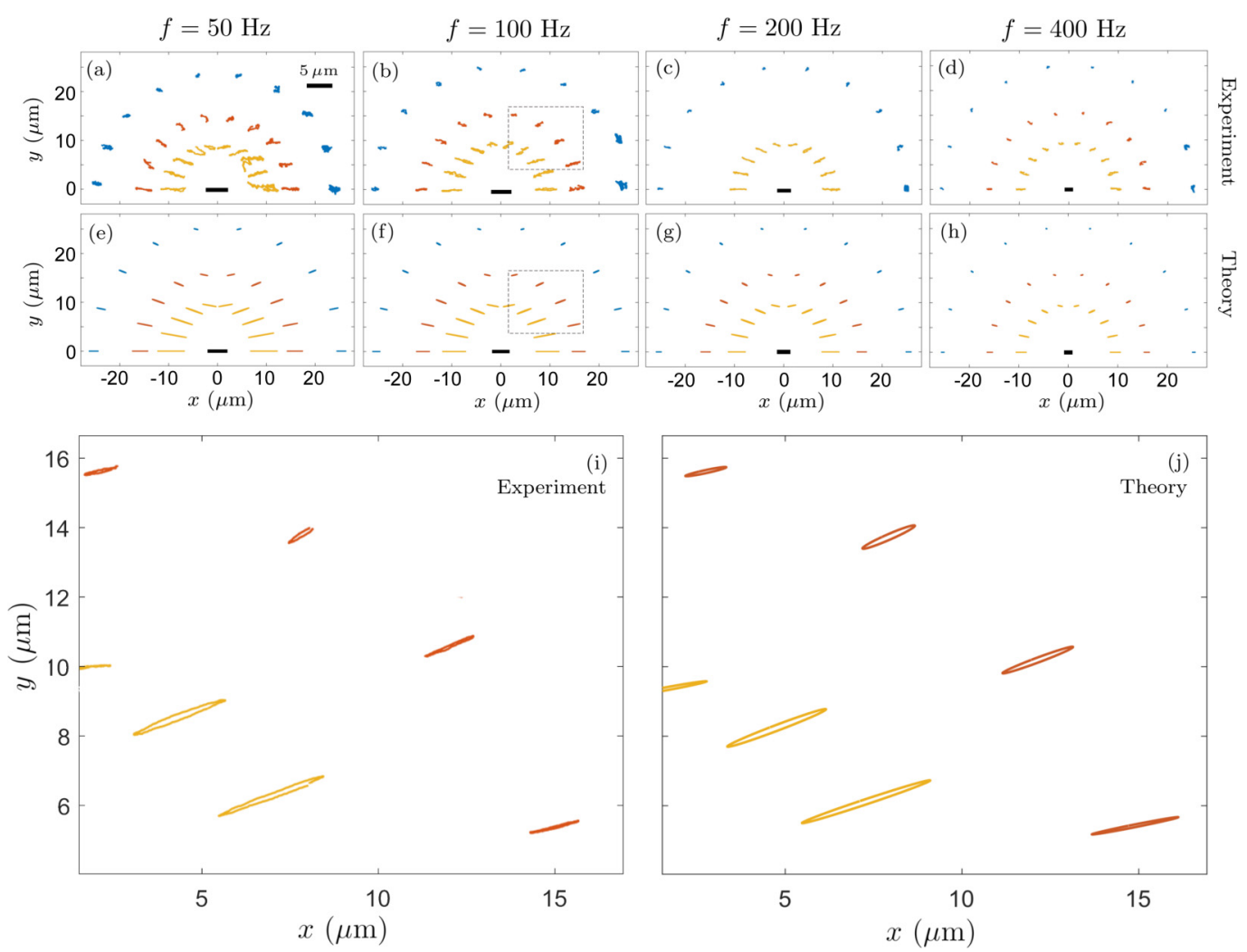

FIG. 3. Oscillatory tracer dynamics, with tracer displacements magnified by a factor 4 , while their equilibrium locations are to the axes scale. (a)-(d) Tracer trajectories over one period. Colors (yellow, red, blue) indicate different radial distances, as in Fig. 1. The peak-to-peak displacement of the central silica bead is indicated by the heavy black line. (e)-(h) Theoretical trajectories using approach given in text. (i), (j) Enlarged view of trajectories in the boxed region shown in (b), (f). (i) shows average cycles from experiments while (j) is simply a zoomed representation of (f). The flat elliptical trajectories, which arise from the phase shift between the components $\xi_{x}^{i j}$ and $\xi_{y}^{i j}$ of the displacement, are accurately captured by the theory.

the prediction of (9) and experiment is excellent. Figure 2(b) shows the accurately sinusoidal displacement of the oscillated particle.

\section{RESULTS AND INTERPRETATION}

\section{A. Tracer dynamics}

Raw trajectories of the probes during a single period of oscillation are shown in Figs. 3(a)-3(d), where for clarity, the probe displacements are magnified by a factor of 4 , while their mean positions are to scale [27]. It is clear that the probe trajectories have a degree of stochasticity superimposed on their background motion. A magnified view of trajectories at $f=100 \mathrm{~Hz}$ that have been averaged by the spectral method described earlier is shown in Fig. 3(i), from which it is apparent that each of the trajectories is an elliptical Lissajous figure whose major and minor axes vary systematically with angular position $\theta$ and distance $R$ from the driven bead.

A quantitative treatment of the probe trajectories and an assessment of the importance of Brownian motion begin with the unsteady velocity field $\boldsymbol{u}(\boldsymbol{r}, t)$ due to a sphere oscillating with velocity $v_{0} \mathrm{e}^{\mathrm{i} \omega t} \hat{\boldsymbol{x}}$. That velocity field satisfies the full Navier-Stokes equations which, if made dimensionless 
by the time $1 / \omega$, a length $L$ and velocity $U$ has two Reynolds numbers [25],

$$
\frac{L^{2} \omega}{v} \boldsymbol{u}_{t^{\prime}}^{\prime}+\frac{U L}{v} \boldsymbol{u}^{\prime} \cdot \nabla^{\prime} \boldsymbol{u}^{\prime}=\nabla^{\prime} \cdot \boldsymbol{\sigma}^{\prime}
$$

where $\sigma^{\prime}$ is the nondimensional hydrodynamic stress. If $L$ is on the scale of the sphere radius $a_{0}$, then $U \sim a_{0} \omega$ and $L^{2} \omega / \nu$ and $U L / v$ are both $\sim a_{0}^{2} \omega / \nu=\left|\alpha_{0}\right|^{2}$, and both the time derivative and nonlinear terms must be considered, but at larger length scales the time derivative dominates the nonlinear term. At the distance $R_{1}$ of the closest tracers, the nonlinear term is already only $\sim 10 \%$ of the inertial term and can be ignored. We have confirmed this by noting the absence of components at frequencies of $2 \omega$ in the power spectrum of the tracer displacements. To find the motion of the tracers we thus examine the solution of the unsteady Stokes equation $\rho_{f} \boldsymbol{u}_{t}=\nabla \cdot \sigma$, together with the continuity equation and boundary conditions $\boldsymbol{u}=\boldsymbol{v}_{0} \mathrm{e}^{\mathrm{i} \omega t} \hat{\boldsymbol{x}}$ at $r=a_{0}$ (particle surface), and $\boldsymbol{u}_{0} \rightarrow 0$ as $r \rightarrow \infty$. The solution, first derived by Stokes [1], can be written as $\boldsymbol{u}=\boldsymbol{u}_{0} \mathrm{e}^{\mathrm{i} \omega t}$, where

$$
\boldsymbol{u}_{0}(\mathbf{r})=v_{0}[A(r) \boldsymbol{I}+B(r) \hat{\boldsymbol{r}} \hat{\boldsymbol{r}}] \cdot \hat{\boldsymbol{x}},
$$

where $\hat{\boldsymbol{r}}$ and $\hat{\boldsymbol{x}}$ are the unit vector along the radial direction and $x$ axis, respectively, and

$$
\begin{aligned}
& A(r)=\frac{3 \alpha_{0}}{2 \rho^{3}}\left[\left(1+\rho+\rho^{2}\right) \mathrm{e}^{\alpha_{0}-\rho}-1-\alpha_{0}-\frac{\alpha_{0}^{2}}{3}\right], \\
& B(r)=\frac{3 \alpha_{0}}{2 \rho^{3}}\left[3+3 \alpha_{0}+\alpha_{0}^{2}-\left(3+3 \rho+\rho^{2}\right) \mathrm{e}^{\alpha_{0}-\rho}\right],
\end{aligned}
$$

where $\rho=(1+i) r / \delta$. For comparison if the sphere were moving along the $x$ axis at a constant speed $v_{0}$ the $\omega \rightarrow 0$ limit of Eq. (11) has the coefficients appropriate to steady flow,

$$
A_{s}=\frac{3 \alpha_{0}}{4 \rho}+\frac{\alpha_{0}^{3}}{4 \rho^{3}}, \quad B_{s}=\frac{3 \alpha_{0}}{4 \rho}-\frac{3 \alpha_{0}^{3}}{4 \rho^{3}},
$$

where these expressions are purely real since $\alpha_{0} / \rho=a_{0} / r$. Note that Stokes' unsteady solution Eq. (11) is only valid for small values of the ratios $\xi_{0} / a_{0}$ or $a_{0} / R$. We consider here that the condition $a_{0} / R \ll 1$ is satisfied even for the first row of tracers, where $a_{0} / R \simeq 0.29$.

We now seek the motion of a probe whose equilibrium position in the oscillating flow is $\mathbf{r}=$ $(R, \theta)$. As we are not referring to any particular tracer, we drop the superscript $i j$. The approach adopted here is a composite one, in which the Lagrangian inertial response of the tracer is computed from the Eulerian flow generated by the central bead. This is valid provided velocity gradients at the probe scale are small, as are the oscillation amplitude relative to the bead radius. Momentum balance for a tracer with velocity $\boldsymbol{v}_{1} \mathrm{e}^{\mathrm{i} \omega t}$ in a fluid with velocity $\boldsymbol{u}_{0} \mathrm{e}^{\mathrm{i} \omega t}$ takes the form $[1,26]$

$$
i \omega \rho_{1} \mathcal{V}_{1} \boldsymbol{v}_{1}=6 \pi \eta a_{1}\left(\Lambda_{1} \boldsymbol{u}_{0}-\Omega_{1} \boldsymbol{v}_{1}\right)
$$

where $\mathcal{V}_{1}=(4 / 3) \pi a_{1}^{3}$ is the volume of the particle and

$$
\Lambda_{1}=1+\alpha_{1}+\frac{\alpha_{1}^{2}}{3}, \quad \Omega_{1}=1+\alpha_{1}+\frac{\alpha_{1}^{2}}{9}, \quad \text { with } \alpha_{1}=(1+i) \frac{a_{1}}{\delta} .
$$

In discussing the motion of the driven particle [cf. Eq. (3)], we noted that the finite-frequency corrections to the drag law were very small; they are even smaller for tracers, whose radii are smaller by a factor of five. It follows that we may safely take $\Lambda_{1}=\Omega_{1}=1$, and thus $i \omega \tau_{d_{1}} \boldsymbol{v}_{1} \simeq\left(\boldsymbol{u}_{0}-\boldsymbol{v}_{1}\right)$, where in parallel with Eq. (4) we define $\tau_{d_{1}}=\left(2 \rho_{1} / 9 \rho\right) a_{1}^{2} / v \sim 5 \times 10^{-8}$ s. This relaxation time is so short relative to the period of driven particle oscillations that we may assume the tracer particle velocity relaxes to that of the fluid instantaneously, and thus the tracer particle velocity is simply

$$
\boldsymbol{v}_{1}=v_{0}[A(r) \boldsymbol{I}+B(r) \hat{\boldsymbol{r}} \hat{\boldsymbol{r}}] \cdot \hat{\boldsymbol{x}} \mathrm{e}^{\mathrm{i} \omega t} .
$$


Combining this result with the response of the driven microsphere, the equations of motion for the tracer displacements, $\dot{\boldsymbol{\xi}}=\boldsymbol{v}_{1}$, integrate to give the tracer motion at $(R, \theta)$,

$$
\xi_{x}(t)=\xi_{0} \Re\left\{\left[A(R)+B(R) \cos ^{2} \theta\right] \mathrm{e}^{\mathrm{i} \omega t}\right\} \quad \text { and } \quad \xi_{y}(t)=\xi_{0} \Re\left\{B(R) \cos \theta \sin \theta \mathrm{e}^{\mathrm{i} \omega t}\right\} .
$$

\section{B. Low-frequency limit of Lissajous figures}

The orbits defined by Eq. (17) are closed, a clear consequence of the linear equation of motion underlying their derivation, and also the use of the Eulerian velocity field at a given coordinate pair $(R, \theta)$ to evaluate the tracer motion. By analogy to the Stokes drift phenomenon in water waves [28], it appears likely that the (small) difference between Eulerian and Lagrangian coordinates during an oscillation cycle of the tracers would lead to (slightly) open orbits, but we defer that analysis to later work. While in direct comparison with experiment we utilize the full expressions in Eq. (17), it is heuristically useful to simplify these results in the regime of low frequencies, when the distances $R_{i}$ of the tracers from the drive sphere are small compared to the viscous penetration depth $\delta$. Thus, expanding Eqs. (12a) and (12b) for $\alpha_{0}, \rho \ll 1$ we find

$$
\begin{aligned}
& A(R) \simeq A_{s}(R)+\alpha_{0}\left(A_{s}(R)-1\right)+\cdots, \\
& B(R) \simeq B_{s}(R)+\alpha_{0} B_{s}(R)+\cdots .
\end{aligned}
$$

Substituting into Eq. (17), assuming as above $R / \delta \ll 1$, we observe that $A_{s}(R)$ and $B_{s}(R)$ are dominated by their Stokeslet contributions $3 a_{0} / 4 R$, and thus

$$
\xi_{x}(t) \simeq \gamma\left(1+\cos ^{2} \theta\right) \cos \left(\omega t+\phi_{x}\right), \quad \xi_{y}(t) \simeq \gamma \sin \theta \cos \theta \cos \left(\omega t+\phi_{y}\right),
$$

where $\gamma(R)=\left(3 a_{0} / 4 R\right) \xi_{0}$, and assuming the phase shifts are small, we find

$$
\phi_{y} \simeq \frac{a_{0}}{\delta} \quad \text { and } \quad \phi_{x} \simeq \phi_{y}-\frac{4 R}{3 \delta} \frac{1}{1+\cos ^{2} \theta} .
$$

Interestingly, while $\phi_{x}$ varies with the polar angle, $\phi_{y}$ does not.

The Lissajous figures associated with Eq. (19) are conic sections [29] and can be rewritten as

$$
\frac{\sin ^{2} \theta}{\left(1+\cos ^{2} \theta\right)^{2}} \xi_{x}^{2}-\frac{2 \tan \theta \cos \phi}{1+\cos ^{2} \theta} \xi_{x} \xi_{y}+\frac{1}{\cos ^{2} \theta} \xi_{y}^{2}-\gamma^{2} \sin ^{2} \theta \sin ^{2} \phi=0,
$$

where the phase shift difference $\phi=\phi_{y}-\phi_{x}$ is

$$
\phi(R, \theta) \simeq \frac{4}{3} \frac{R}{\delta} \frac{1}{1+\cos ^{2} \theta} .
$$

We note parenthetically that the limit $a_{0} \rightarrow 0$ in Eq. (11) would correspond formally to an oscillating point force [25], known as an "oscillet" and used in recent work on the dynamics of beating flagella [17]. The precise limiting procedure involves setting $a_{0} \rightarrow 0$ inside the square brackets of Eqs. (12a) and (12b), while letting the prefactor velocity $v_{0} \rightarrow \infty$ such that the product $6 \pi \mu a_{0} v_{0} \rightarrow F$, where $F$ is the magnitude of the oscillating force. In that limit, the $y$-component phase shift $\phi_{y}$ in Eq. (20) vanishes, but $\phi_{x}$ is otherwise unchanged, so the difference $\phi$ is still given by Eq. (22).

Returning to the Lissajous figures, we note that Eq. (21) is in the standard form $\mathcal{A} \xi_{x}^{2}+$ $\mathcal{B} \xi_{x} \xi_{y}+\mathcal{C} \xi_{y}^{2}+\mathcal{F}=0$ of conic sections, which are in this cases ellipses since $\mathcal{D} \equiv \mathcal{B}^{2}-4 \mathcal{A} C=$ $-4\left[\tan \theta \sin \phi /\left(1+\cos ^{2} \theta\right)\right]^{2}<0$. A standard analysis shows that the major axis of the ellipse is tilted with respect to the $x$ axis by an angle $\psi$ satisfying $\tan 2 \psi=\mathcal{B} /(\mathcal{A}-\mathcal{C})$. As $\psi$ varies with $\cos \phi$, corrections to the $\phi=0$ limit are $\mathcal{O}\left[(R / \delta)^{2}\right]$, so $\psi$ is well-approximated by the tilt angle of a steady stokeslet,

$$
\psi_{s}=\tan ^{-1}\left(\frac{\sin \theta \cos \theta}{1+\cos ^{2} \theta}\right) .
$$


TABLE I. Physical quantities for experiments in water in a convenient system of units.

\begin{tabular}{lclc}
\hline \hline Symbol & Definition & & Quantity \\
\hline$v$ & $\eta / \rho_{w}$ & Kinematic viscosity of water & $10^{6} \mu \mathrm{m}^{2} \mathrm{~s}^{-1}$ \\
$\delta$ & $(2 \nu / \omega)^{1 / 2}$ & Viscous penetration depth & $28-80 \mu \mathrm{m}$ \\
$a_{0}$ & & Radius of driven microsphere & $2.77 \mu \mathrm{m}$ \\
$a_{1}$ & & Radius of tracer microspheres & $0.505 \mu \mathrm{m}$ \\
$D_{1}$ & $\mathrm{k}_{B} \mathrm{~T} / 6 \pi \eta a_{1}$ & Diffusion constant tracer microspheres & $0.43 \mu \mathrm{m}^{2} \mathrm{~s}^{-1}$ \\
$\rho_{0}$ & & Density of driven microsphere & $2.65 \times 10^{3} \mathrm{~kg} \mathrm{~m}^{-3}$ \\
$\rho_{1}$ & & Density of tracer microspheres & $1.05 \times 10^{3} \mathrm{~kg} \mathrm{~m}^{-3}$ \\
$R_{i}$ & $i=1,2,3$ & Unperturbed radial distances of tracers & $9.5,15.8,25.4 \mu \mathrm{m}$ \\
$\theta_{j}$ & $(j-1) \pi / 9$ & Unperturbed angular position of tracers & $j=1, \ldots, 10$ \\
$k$ & & Optical trap stiffness & $50 \mathrm{pN} \mu \mathrm{m}^{-1}$ \\
$\omega$ & $2 \pi f$ & Oscillation frequency of driven microsphere & $2 \pi \times(50-400 \mathrm{~Hz})$ \\
$\zeta_{\text {trap }}$ & & Oscillation amplitude of optical trap & $2.15 \mu \mathrm{m}$ \\
$\xi_{0}(\omega)$ & $\left|\zeta_{0}(\omega)\right|$ & Oscillation amplitude of driven microsphere & $(0.4-1) \times \xi_{\text {trap }}$ \\
$\xi^{i j}$ & & Lagrangian displacement of sphere at $\left(R_{i}, \theta_{j}\right)$ & $<0.4 \times \xi_{0}$ \\
$\chi^{i j}$ & $\max \left(\xi_{x}^{i j} / \xi_{0}\right)$ & Scaled maximum Lagrangian $x$ displacement of sphere at $\left(R_{i}, \theta_{j}\right)$ & $<0.4$ \\
$\phi$ & $\phi_{y}-\phi_{x}$ & Relative phase lag of $x$ and $y$ components of tracers & $\delta \pi / 2$ \\
\hline \hline
\end{tabular}

The fundamental signature of unsteadiness in the present experiment is the elliptical form of the tracer orbits. From the general expression for the semimajor and semiminor axes of ellipses,

$$
a^{2}, b^{2}=\frac{2 \mathcal{F}}{\mathcal{D}}\left\{\mathcal{A}+\mathcal{C} \pm\left[(\mathcal{A}+\mathcal{C})^{2}+\mathcal{D}\right]^{1 / 2}\right\}
$$

where the $+(-)$ sign refers to $a(b)$, we obtain the remarkably simple asymptotic results,

$$
\frac{a}{\xi_{0}}=\frac{3 a_{0}}{4 R} \frac{1+\cos ^{2} \theta}{\cos \psi_{s}}+\cdots, \quad \text { and } \quad \frac{b}{\xi_{0}}=\frac{a_{0}}{\delta}\left|\sin \psi_{s}\right|+\cdots .
$$

The results in Eq. (25) are the heuristic relationships we sought. They show that to leading order at low frequencies the semimajor axis $a$ is given simply by the motion of the driven particle, projected to its position via the stokeslet contribution, while the semiminor axis $b$ is nonzero only to the extent that the viscous penetration length itself is not infinite. The aspect ratios of the ellipses simply reflect the phase shift; $b / a=(\phi / 2)\left|\sin 2 \psi_{s}\right|$, and in the steady limit the ellipses degenerate into lines. For later reference we note if we include the leading unsteady corrections, then the normalized $x$-component of the tracer displacements can be written as

$$
\chi \equiv \frac{\max \left\{\xi_{x}\right\}}{\xi_{0}}=\chi_{s}-\frac{a_{0}}{\delta}+\cdots, \quad \text { with } \quad \chi_{s}=\frac{3 a_{0}}{4 R}\left(1+\cos ^{2} \theta\right) .
$$

\section{Role of thermal fluctuations}

Before analyzing the tracer trajectories in detail, we use the particle trajectories to quantify the competition between the deterministic forcing of the tracer particles and thermally driven Brownian motion. As in previous discussions of this issue [15], a useful metric with which to assess these effects is the ratio of the maximum deterministic displacement due to the oscillating fluid (the major axis of the ellipse) to the average Brownian displacement over half an oscillation period. This is essentially the square root of a Péclet number,

$$
\sqrt{\mathrm{Pe}}=\frac{2 a}{\left(2 \pi D_{1} / \omega\right)^{1 / 2}} \simeq \frac{3}{2 \sqrt{\pi}}\left(\frac{a_{0}}{R}\right)\left(\frac{\xi_{0}}{\delta}\right) \sqrt{\frac{\nu}{D_{1}}} \frac{1+\cos ^{2} \theta}{\cos \psi_{s}} .
$$

Here, $D_{1}=k_{B} T / 6 \pi \eta a_{1} \sim 0.4 \mu \mathrm{m}^{2} / \mathrm{s}$ (Table I) is the diffusion constant of the tracers, with $k_{B}$ the Boltzmann constant and $T=298 \mathrm{~K}$ the absolute temperature. The last relation in Eq. (27) is 

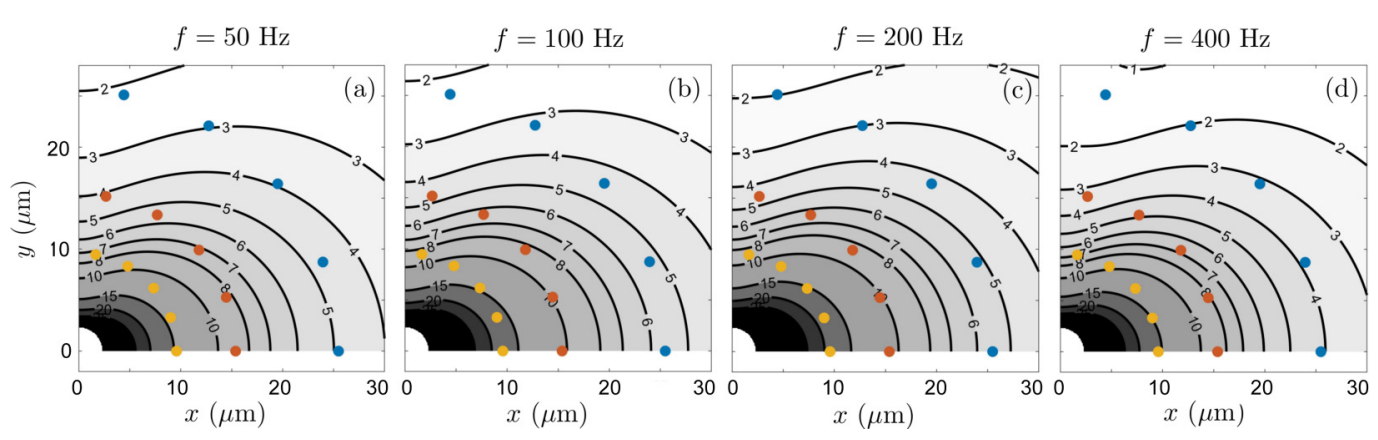

FIG. 4. Significance of thermal fluctuations. (a)-(d) Contour plots of the $\sqrt{\text { Pe }}$ [Eq. (27)] in physical space, with the locations of the probe microspheres superimposed, at the four driving frequencies.

obtained using the asymptotic results above and displayed to emphasize it is the product of three dimensionless ratios. The factor $v / D_{1}$ is a Schmidt number Sc for the tracer particles and is very large $\left(\sqrt{\mathrm{Sc}} \simeq 1.6 \times 10^{3}\right)$, but its contribution to Pe is attenuated by the two small factors $a_{0} / R$ and $\xi_{0} / \delta$, each on the order of 0.1 . The frequency dependence of $\sqrt{\mathrm{Pe}}$ is relatively weak by virtue of the counteracting trends of $\xi_{0} \sim \omega^{-1}$ and $\delta \sim \omega^{-1 / 2}$. Contour plots of Eq. (27) in the first quadrant of physical space where the tracers reside are shown in Fig. 4, in which the semimajor axis has been computed with the full unsteady velocity field given in Eqs. (11), (12a), and (12b). From these results we see that advective contributions dominate diffusion $(\mathrm{Pe}>1)$ at all frequencies for the innermost spheres, while the two become comparable for the outermost spheres, consistent with the qualitative appearance of the trajectories.

\section{Comparison with theory}

A first test of the theoretical analysis of the trajectories involves plotting the ellipses from Eq. (21) in the $x, y$ plane. These are shown in Figs. 3(e)-3(h), magnified by a factor 4 to be consistent with all the plots of the figure. In addition, the boxed portion of Fig. 3(f) is expanded in Fig. 3(j). In comparison, Fig. 3(i) displays the average oscillation of a few tracers at the same locations, from experiments, showing a good match with the theory. Alternatively, Fig. 5 shows two basic geometrical features of the elliptical tracer trajectories, their orientation and major axis, each expected to be dominated by their steady contributions. The orientation angle in Fig. 5(a) agrees well with the steady angle $\psi_{s}$ in Eq. (25), and the semimajor axis [Fig. 5(a)] is likewise well described by the leading order relation in Eq. (23).

Focusing on the displacements along the same $(x)$ axis as the driven microsphere, Fig. 6 summarizes the results for the amplitudes and phase shifts of the tracers. In Figs. 6(a)-6(d) we plot the normalized component of the displacement as defined in Eq. (26),

$$
\chi^{i j}=\frac{\max \left\{\xi_{x}^{i j}\right\}}{\xi_{0}},
$$

for experiments (symbols) and theory (lines). In the experiments, the relative amplitude and phase compared to the driven bead are measured from the fast Fourier transform (FFT) of the $x(t)$ data of each tracer, by identifying the amplitude (respectively, the phase) from the peaks in the magnitude (phase) plots of the FFT for the tracers and dividing by the magnitude (or subtracting the phase) from the FFT of the driven bead. At any given frequency the agreement between the data and the steady theory (shown by dashed lines) is best for those tracers closest to the driven particle and progressively decreases for more distant probes, while at any given radius the agreement with the steady theory worsens at higher frequencies. For example, the deterministic component of the displacement is overestimated by about $20 \%$ for $f=50 \mathrm{~Hz}$, and up to $100 \%$ (for $f=400 \mathrm{~Hz}$ ) 

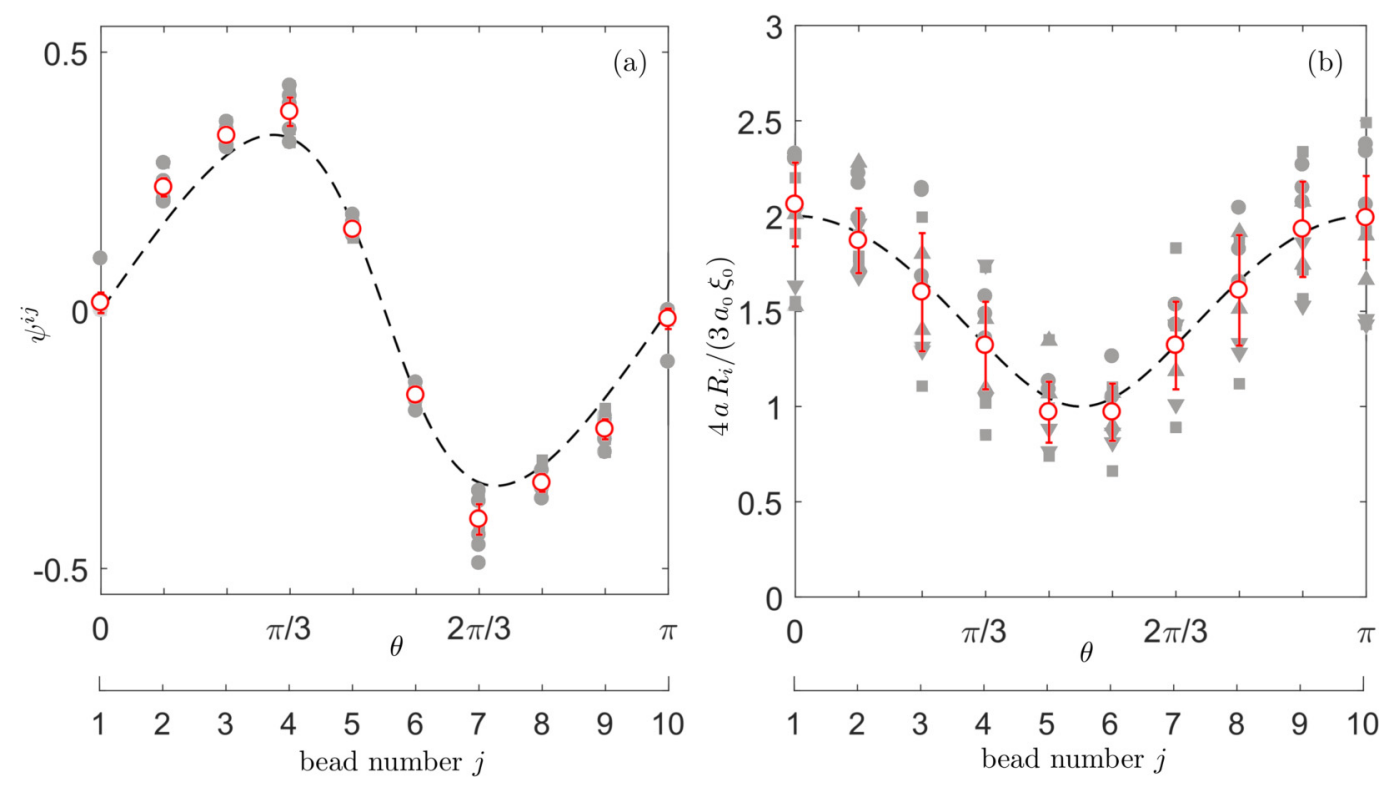

FIG. 5. Orientation and size of elliptical tracer trajectories. (a) Tilt angle of the major axis of elliptical Lissajous figures as a function of mean angular position relative to driving axis. Gray symbols represent the individual data points at the four experimental frequencies $(f=50,100,200$, and $400 \mathrm{~Hz})$ and three radii $(R=9.5,15.8$, and $25.4 \mu \mathrm{m})$ at a given angular position $\theta_{i}$. Open red circles denote the mean values of each of those measurements at a given $\theta$ and their standard deviations. Dashed line is the steady tilt angle Eq. (23). (b) As in panel (a), but for the semimajor axis of the ellipses. Dashed line is the low-frequency limit Eq. (25).
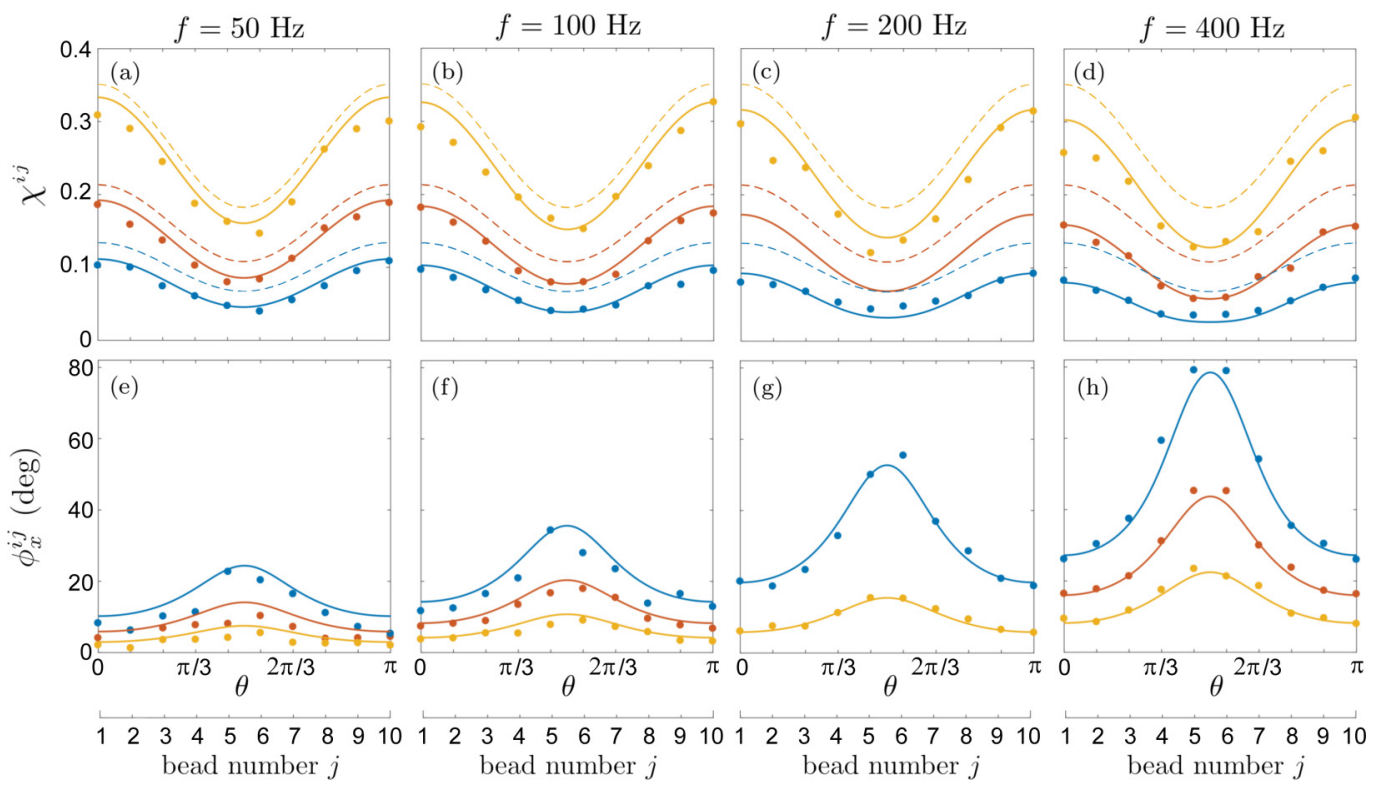

FIG. 6. Dynamics of the passive tracers. (a)-(d) Normalized maximum displacements (solid circles), and their theoretical counterpart $\chi$ (solid lines). Dashed line show displacements calculated within steady Stokes equation. (e)-(h) Phase shifts between responses of the probes and the harmonic motion of driving bead (solid circles) and theoretical counterpart (solid lines). Colors (yellow, red, blue) indicate different radial distances, as in Fig. 1. 

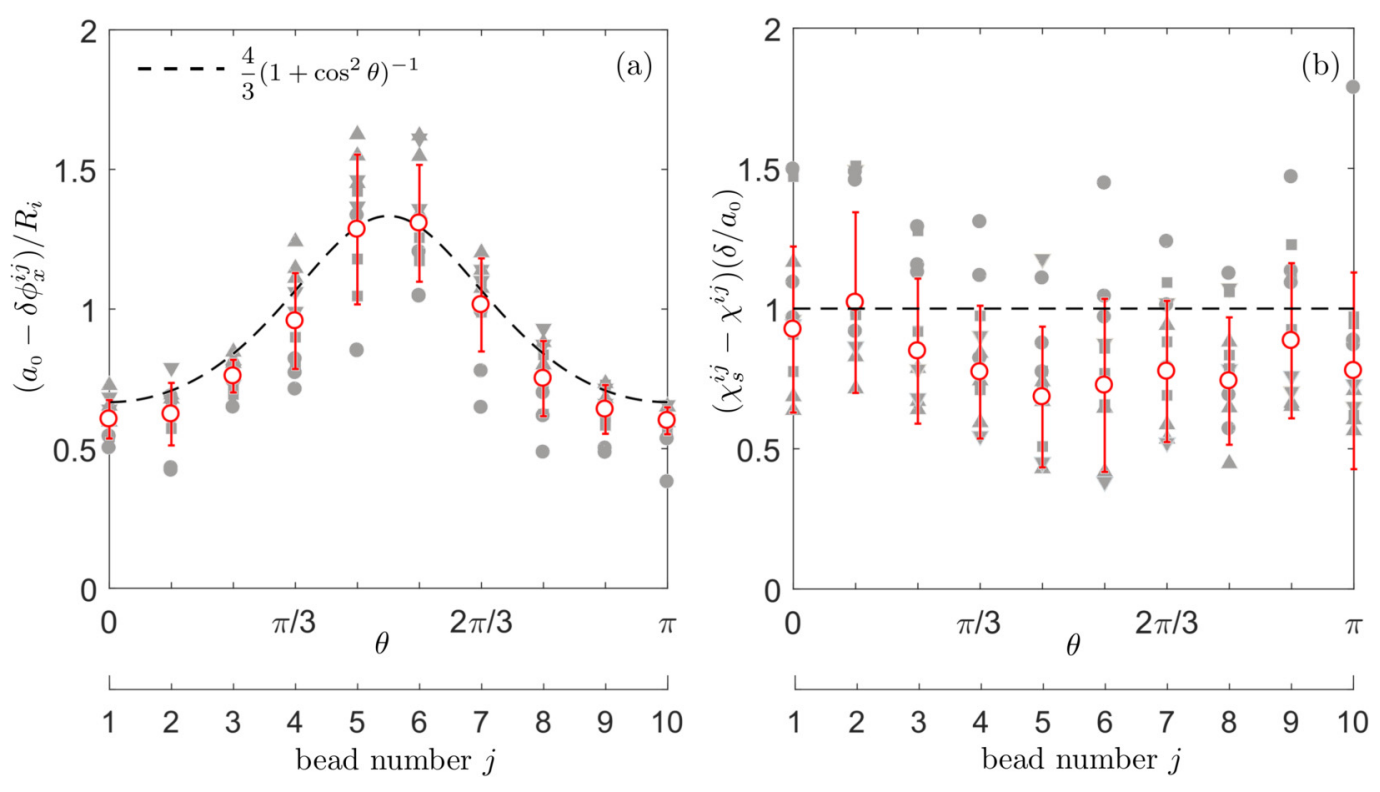

FIG. 7. Test of the low-frequency scaling. (a) Rescaled phase shift as a function of angle, using same symbol motif as in Fig. 5. Dashed line is the scaling result Eq. (29). (b) As in panel (a) but for the oscillation amplitude, with dashed line from Eq. (29) to leading order. There are no adjustable parameters in panel (a) or panel (b).

for the most remote ones, i.e., at a distance $R_{3}$ from the origin. Both of these trends are fully consistent with the relevant measure of unsteadiness being $R / \delta$. In Figs. $6(\mathrm{e})-6(\mathrm{~h})$ we show the the experimental phase shift $\phi_{x}^{i j}$ between the tracers and the active particle. The magnitude and angular dependence are both accurately captured by the unsteady theory. At the very highest frequency used, the phase shifts of the most distant probes located close to the $y$ axis-at $\left(R_{3}, \theta_{5}\right)$ and $\left(R_{3}, \theta_{6}\right)$-are very large; the probes are almost in quadrature with the forcing. We see from these results that despite a large displacement of the central bead (which is of the same order as its radius) and a direct use of the Eulerian form of the viscous unsteady flow $\boldsymbol{u}_{0}$, the agreement between theory and experiment is very good, with a maximum relative error of $4 \%$ overall.

As a final test of the unsteady theory, we ask whether the data in Fig. 6 are consistent with the predicted leading-order low-frequency limits in the sense of a data collapse. Focusing on the same $x$ component of the phase shift and amplitude, the analysis in Eqs. (20) and (22) can be written the scaling forms for the phase and amplitude,

$$
\frac{a_{0}-\delta \phi_{x}}{R}=\frac{4}{3} \frac{1}{1+\cos ^{2} \theta}, \quad \text { and } \quad \frac{\delta}{a_{0}}\left(\chi_{s}-\chi\right)=1+\cdots .
$$

Figure 7 shows good agreement in both cases, especially for the phase shift. In the case of the amplitude, the terms beyond leading order involve the same factor $1+\cos ^{2} \theta$ seen in the phase, with an amplitude that is nonuniversal (i.e., one that depends on the radii $R_{i}$ ); this can be seen in the small but systematic deviations from the limiting value of unity in Fig. 7(b).

\section{DISCUSSION}

We have shown here how optical trapping and particle-tracking techniques allow for a precise microscale test of the theory of unsteady Stokes flows. At the scale of colloidal particles, and with oscillation frequencies in the range found in biological systems, the simplifications arising from lack of inertial effects on particle motion and corrections to the Stokes drag law allow for a simple 
and compact picture of the particle orbits. The regime of sizes and frequencies explored is also such that Brownian motion makes only a modest contribution to the tracer dynamics, with an effective Péclet number generally exceeding unity. Our experimental results show that tracer particles move on simple elliptical orbits even in a regime with very large phase shifts, in quantitative accord with a low-frequency analysis. These experimental observations would be difficult to reproduce by conventional particle imaging techniques which are based on obtaining an Eulerian velocity map from correlation functions of small tracer displacements.

As outlined in the introduction, one clear motivation for the present study is provided by the evidence that unsteady effects are present during the collective beating of eukaryotic cilia and flagella. It is an open question as to whether these effects actually control synchronization. Two features of the present work will likely bear on this issue; the angular dependence of the phase shift and the elliptical orbits themselves. The former dictates the strength of the lateral coupling between cilia along a tissue, while the latter represents vorticity created by the driven particle that may be relevant to wave propagation. It is important to note that in the many cases in which metachronal waves occur there is a nearby underlying no-slip surface-the cell wall of a ciliate, or the tissue surface of an ciliated epithelium-whose presence can not be ignored. Indeed, a recent study of synchronization in arrays of "colloidal oscillators" [14], microspheres moved along periodic orbits by optical traps, show that surface proximity can profoundly affect the collective dynamics that they exhibit. Thus, a natural next step is the study of model unsteady flows near no-slip surfaces [30].

\section{ACKNOWLEDGMENTS}

This work was supported in part by ERC Consolidator Grant No. 682754 (E.L.), ERC PoC Grant CellsBox (P.C. and J.K.), Wellcome Trust Investigator Award No. 207510/Z/17/Z, Established Career Fellowship No. EP/M017982/1 from the Engineering and Physical Sciences Research Council, and the Marine Microbiology Initiative of the Gordon and Betty Moore Foundation, Grant No. 7523 (R.E.G.).

[1] G. G. Stokes, On the effect of the internal friction of fluids on the motion of pendulums, Trans. Cambridge Philos. Soc. 9(2), 8 (1851).

[2] C. Brennen, An oscillating boundary-layer theory for ciliary propulsion, J. Fluid Mech. 65, 799 (1974).

[3] R. E. Goldstein, Green algae as model organisms for biological fluid dynamics, Annu. Rev. Fluid Mech. 47, 343 (2015).

[4] D. R. Brumley, M. Polin, T. J. Pedley, and R. E. Goldstein, Hydrodynamic Synchronization and Metachronal Waves on the Surface of the Colonial Alga Volvox Carteri, Phys. Rev. Lett. 109, 268102 (2012).

[5] D. R. Brumley, M. Polin, T. J. Pedley, and R. E. Goldstein, Metachronal waves in the flagellar beating of Volvox and their hydrodynamic origin, J. R. Soc., Interface 12, 20141358 (2015).

[6] S. Gueron, K. Levit-Gurevich, N. Liron, and J. J. Blum, Cilia internal mechanism and metachronal coordination as the result of hydrodynamical coupling, Proc. Natl. Acad. Sci. USA 94, 6001 (1997); S. Gueron and K. Levit-Gurevich, Energetic considerations of ciliary beating and the advantage of metachronal coordination, ibid. 96, 12240 (1999).

[7] J. Elgeti and G. Gompper, Emergence of metachronal waves in cilia arrays, Proc. Natl. Acad Sci. USA 110, 4470 (2013).

[8] B. Qian, H. Jiang, D. A. Gagnon, K. S. Breuer, and T. R. Powers, Minimal model for synchronization induced by hydrodynamic interactions, Phys. Rev. E 80, 061919 (2009).

[9] M. C. Lagomarsino, P. Jona, and B. Bassetti, Metachronal waves for deterministic switching twostate oscillators with hydrodynamic interaction, Phys. Rev. E 68, 021908 (2003).

[10] C. Wollin and H. Stark, Metachronal waves in a chain of rowers with hydrodynamic interactions, Eur. Phys. J. E 34, 42 (2011). 
[11] A. Vilfan and F. Jülicher, Hydrodynamic Flow Patterns and Synchronization of Beating Cilia, Phys. Rev. Lett. 96, 058102 (2006).

[12] P. Lenz and A. Ryskin, Collective effects in ciliar arrays, Phys. Biol. 3, 285 (2006).

[13] T. Niedermayer, B. Eckhardt, and P. Lenz, Synchronization, phase locking, and metachronalwave formation in ciliary chains, Chaos 18, 037128 (2008).

[14] D. R. Brumley, N. Bruot, J. Kotar, R. E. Goldstein, P. Cicuta, and M. Polin, Long-range interactions, wobbles and phase defects in chains of model cilia, Phys. Rev. Fluids 1, 081201(R) (2016).

[15] K. C. Leptos, J. S. Guasto, J. P. Gollub, A. I. Pesci, and R. E. Goldstein, Dynamics of Enhanced Tracer Diffusion in Suspensions of Swimming Eukaryotic Microorganisms, Phys. Rev. Lett. 103, 198103 (2009).

[16] D. Wei, P. G. Dehnavi, M.-E. Aubin-Tam, and D. Tam, Is the Zero Reynolds Number Approximation Valid for Ciliary Flows? Phys. Rev. Lett. 122, 124502 (2019).

[17] D. Wei, P. G. Dehnavi, M.-E. Aubin-Tam, and D. S. W. Tam, Measurements of the unsteady flow field around beating cilia, J. Fluid Mech. 915, A70 (2021).

[18] P. G. Dehnavi, D. Wei, M.-E. Aubin-Tam, and D. S. W. Tam, Optical tweezers-based velocimetry: A method to measure microscale unsteady flows, Exp. Fluids 61, 202 (2020).

[19] N. Bruot and P. Cicuta, Realizing the physics of motile cilia synchronization with driven colloids, Annu. Rev. Condens. Matter Phys. 7, 323 (2016).

[20] P. Cicuta, The use of biophysical approaches to understand ciliary beating, Bioch. Soc. Trans. 48, 221 (2020).

[21] D. R. Brumley, K. Y. Wan, M. Polin, and R. E. Goldstein, Flagellar synchronization through direct hydrodynamic interactions, eLife 3, e02750 (2014).

[22] J. Kotar, L. Debono, N. Bruot, S. Box, D. Phillips, S. Simpson, S. Hanna, and P. Cicuta, Optimal Hydrodynamic Synchronization of Colloidal Rotors, Phys. Rev. Lett. 111, 228103 (2013).

[23] A. Maestro, N. Bruot, J. Kotar, N. Uchida, R. Golestanian, and P. Cicuta, Control of synchronization in models of hydrodynamically coupled motile cilia, Commun. Phys. 1, 28 (2018).

[24] K. C. Neuman and S. M. Block, Optical trapping, Rev. Sci. Instrum 75, 2787 (2004).

[25] S. Kim and S. J. Karrila, Microhydrodynamics: Principles and Selected Applications (Courier, Chelmsford, 2013).

[26] P. Mazur and D. Bedeaux, A generalization of Faxen's theorem to nonsteady motion of a sphere through an incompressible fluid in arbitrary flow, Physica 76, 235 (1974).

[27] The data for the red tracers $\left(R_{2}, f=200 \mathrm{~Hz}\right)$ in Figs. 3(c) and 3(g) are not available due to unforeseen data corruption on the hard-drive. The missing trajectories do not affect the overall trend and results presented here.

[28] G. G. Stokes, On the theory of oscillatory waves, Trans. Cambridge Philos. Soc. 8, 441 (1847).

[29] J. Wallis, De sectionibus conicis nova methodo expositis tractatus (Oxonii: Typis Leon Lichfield Academiæ typographi, impensis Tho. Robinson, 1655).

[30] I. Fouxon and A. Leshansky, Fundamental solution of unsteady Stokes equations and force on an oscillating sphere near a wall, Phys. Rev. E 98, 063108 (2018). 